\title{
Las herramientas de consulta en la enseñanza- aprendizaje del inglés: ¿diccionarios monolingües de aprendizaje o diccionarios generales bilingües?
}

\author{
M. ${ }^{a}$ Teresa Ortego Antón \\ Universidad de Valladolid \\ tortego@lesp.uva.es
}

Resumen: El inglés se ha convertido en la lengua vehicular del saber especializado y su aprendizaje es una tarea imprescindible para desenvolverse en la sociedad actual. Además, el léxico especializado ha pasado de pertenecer a un grupo restringido de expertos a formar parte del acervo lingüístico de un hablante con un nivel cultural medio. En este contexto, una de las herramientas a la que acuden los aprendices de esta lengua para solventar los obstáculos que surgen durante el trasvase interlingüístico se corresponde con los diccionarios electrónicos, tanto generales bilingües como monolingües de aprendizaje. Por nuestra experiencia como docentes de lenguas, consideramos que las obras mencionadas ofrecen una cobertura parcial y desigual del léxico especializado, razón por la que estimamos pertinente realizar un estudio del tratamiento conferido a este léxico en los diccionarios generales bilingües y en los diccionarios de aprendizaje. Con un análisis de una muestra de léxico especializado del campo de la informática e Internet, pretendemos obtener un diagnóstico lo más ajustado posible de la realidad, de manera que podamos valorar como docentes de lenguas extranjeras la eficacia o no de dichas obras a la hora de responder a las necesidades de nuestros estudiantes. En última instancia, redundará en pautas fidedignas a la hora de implementar el diseño y uso de estas obras.

Palabras clave: diccionario monolingüe de aprendizaje, diccionario general bilingüe, enseñanza-aprendizaje, léxico especializado, lengua extranjera. 
Abstract: English has been the language used to communicate specialised knowledge. Its learning is an essential task in order to get on the globalised world. In addition, specialised vocabulary, which used to be exclusively for experts, is now also of interest to middlebrow language users. In this background, general bilingual dictionaries as well as learner's dictionaries have become one of the most used electronic tools by language learners. However, we consider that these works offer a partial coverage of specialised vocabulary. Hence, a study about a sample of computing and Internet terms gathered in general bilingual dictionaries and learner's dictionaries could provide us an accurate diagnosis in order to determine whether our students' needs are satisfied or not and to improve the design and use of these tools.

Keywords: learner's dictionary, general bilingual dictionary, teaching-learning process, specialised vocabulary, foreign language. 


\section{El inglés, lingua franca en la sociedad actual}

La lengua inglesa ha cobrado importancia en la difusión del conocimiento experto desde el siglo xix. Dicha relevancia se hizo todavía más imponente con la fuga de cerebros hacia América a partir de los años 30 del siglo xx.

Desde entonces científicos de diferentes nacionalidades se han dedicado a cultivar las áreas de especialidad utilizando la lengua inglesa, de manera que el inglés se ha convertido en la lengua de comunicación e intercambio en todos los niveles y esferas de la sociedad (Gutiérrez Rodilla, 1998: 81).

Conscientes de la popularidad de la lengua inglesa como vehículo del saber especializado en un contexto globalizado, la sociedad de nuestros días considera que su aprendizaje es una tarea imprescindible para desenvolverse en el mundo actual.

En consecuencia, las entidades gubernamentales incluyen esta lengua en los currículos de enseñanza, desde Educación Infantil hasta la universidad. No obstante, el aprendizaje del inglés todavía es una tarea pendiente para gran parte de la población adulta hispanohablante, a pesar de que las empresas cada vez con más frecuencia requieren que los trabajadores acrediten conocimientos en esta lengua.

En este contexto las editoriales no se han mostrado ajenas a este fenómeno y han publicado infinidad de materiales destinados al aprendizaje de la lengua inglesa, basados en diferentes metodologías y orientados a la gran variedad de perfiles que aglutinan a los estudiantes de lengua inglesa.

Entre los recursos que asisten a los estudiantes durante la codificación y descodificación de los mensajes, la herramienta tradicionalmente más utilizada se corresponde con los diccionarios, ya sean monolingües o bilingües. Sin embargo, en las últimas décadas hemos sido testigos de la aparición de nuevas tipologías de diccionarios, como los diccionarios bilingualizados, o incluso de programas como los writing assistants o las bases de datos léxicas, que asisten a los aprendices durante las actividades de producción.

Por tanto, conscientes de la variedad de recursos al alcance de los estudiantes de lengua inglesa, en el presente trabajo optamos por centrar la atención en el papel de los diccionarios generales bilingües y de los diccionarios monolingües de aprendizaje como herramientas para dar respuesta a los obstáculos que surgen durante el transvase interlingüístico. 


\section{Los diccionarios electrónicos, herramienta imprescindible en el aprendizaje de una lengua extranjera}

En la literatura lexicográfica han sido muchos los autores que han definido y caracterizado los diccionarios, así como sus funciones, que están determinadas por las necesidades de los usuarios (Béjoint, 1981; Hartmann, 1989; Bergenholtz y Tarp, 2002). En este sentido, en un contexto de enseñanza-aprendizaje, las tareas llevadas a cabo por los estudiantes pueden ser activas o relacionadas con la codificación de un mensaje. Dentro de esta función se incluyen actividades como la composición, la expresión oral y la traducción de lengua materna a lengua extranjera. Por otro lado, los estudiantes de lenguas extranjeras también pueden acudir al diccionario para dar solución a tareas pasivas, en las que el objetivo es descodificar un mensaje. Entre las actividades cubiertas por esta función destaca la comprensión oral y escrita así como la traducción de lengua extranjera a lengua materna (Fuertes Olivera y Arribas Baño, 2008: 3).

Tradicionalmente, para resolver las dudas que aparecían en el trascurso del trasvase interlingüístico, los estudiantes de lenguas solían acudir a los diccionarios generales bilingües. Hartmann y James (1998: 230) definen esta tipología de obras de la siguiente manera:

A type of dictionary which relates the vocabulary of two languages together by means of translation equivalents, in contrast to the monolingual dictionary, in which explanations are provided in one language. This is at one its greatest advantage and disadvantage. By providing lexical equivalents, the bilingual dictionary helps language learners and translators to read and create texts in a foreign language. However, finding suitable lexical equivalents is a notoriously difficult task, especially in pairs of languages with different.

No obstante, conviene apuntar que en las últimas décadas han sido muchas las voces (McArthur, 1998, Cowie, 1999; Stein, 2002; Tono, 1996; Granger, 1994; Nesi, 1999, 2000; Tarp, 2006; Granger y Paquot, 2010) que promulgan la utilización de diccionarios monolingües adaptados a las necesidades de los estudiantes de lenguas, puesto que pueden considerarse como la herramienta intermedia entre el diccionario bilingüe, que suele emplearse en los niveles iniciales de aprendizaje, y el diccionario monolingüe, cuyo uso suele resultar demasiado complicado para los aprendices principiantes de lengua extranjera.

En este sentido, hay que remontarse a la década de los años 30 del siglo pasado para situar el comienzo de los diccionarios de aprendizaje. Harold Palmer, Michael West y Albert S. Hornby, impulsados por la creciente importancia del 
inglés como lengua de comunicación internacional y la correspondiente necesidad de que millones de personas estudiaran y aprendieran dicha lengua, emprendieron el camino hacia lo que más adelante se conocería como diccionarios de aprendizaje (Tarp, 2006: 296). De hecho, estas obras se convirtieron en una tipología de diccionarios en la segunda mitad del siglo xx, debido al auge de la enseñanza del inglés como lengua extranjera. Además, los avances que se han producido tanto en metalexicografía como en lingüística de corpus han consolidado el éxito de estas obras, que se caracterizan por incluir una serie de información que los distingue de otros tipos de diccionarios:

ESL dictionaries include some of the features of foreign-language dictionaries, such as providing more information than native-speaker dictionaries on grammar, phrasal verbs, and idiomatic usages. May ESL dictionaries use a simplified, controlled vocabulary for their definitions, generally limited to 2,000 or 3,000 words (but often including derivative forms as well, thus stretching the total). ESL dictionaries devote a substantial amount of space to exemplifying definitions with illustrative phrases or sentences [...] and are designed to enable the foreign learner to produce utterances in English (the encoding function), not just to comprehend them (the decoding function) (Landau, 2001: 16-17).

Por otro lado, el conocimiento especializado ha pasado, de ser patrimonio exclusivo de los expertos, a interesar también a los usuarios de una lengua con un nivel cultural medio, fruto de la democratización de la educación y de la difusión que llevan a cabo los medios de comunicación.

En consecuencia, conscientes de la relevancia que los diccionarios bilingües y los diccionarios monolingües de aprendizaje han adquirido como herramientas para dar respuesta a los obstáculos que surgen durante la comunicación interlingüística, así como del impacto que las unidades léxicas que hacen referencia a conceptos especializados tienen en la lengua general, consideramos que actualmente las obras existentes ofrecen una cobertura parcial y desigual de las principales unidades léxicas especializadas utilizadas por los hablantes de un lengua con un nivel cultural medio.

Por tanto, estimamos pertinente realizar un estudio contrastivo del tratamiento conferido al léxico especializado en los diccionarios generales bilingües y en los diccionarios monolingües de aprendizaje. Dicho estudio nos permitirá obtener un diagnóstico más ajustado de la realidad, para valorar como docentes de lenguas extranjeras su eficacia o no a la hora de responder a las necesidades de nuestros estudiantes y, en última instancia, redundará en unas pautas fidedignas a la hora de implementar el diseño y uso de estas obras. 


\section{Delimitación del objeto de análisisł el léxico de la informática e Internet}

El estudio del tratamiento conferido a la totalidad de las unidades léxicas especializadas registradas en estas obras es una tarea que supera los límites temporales y humanos de los que disponemos, además de ser considerada una labor casi imposible por varios autores (Thoiron, 1998; Rodríguez Reina, 2002). En consecuencia, a partir de la consideración de la metodología expuesta en trabajos previos (Roberts, 2004; Josselin, 2005) estimamos oportuno centrar el estudio en un determinado campo del saber, la informática e Internet.

Actualmente las aplicaciones informáticas vertebran la práctica totalidad de los campos de especialidad de nuestra sociedad, hasta el punto de que sus transformaciones inciden en gran medida en el avance de la mayoría de las áreas del saber humano.

De hecho, dicho carácter interdisciplinar hará posible que el estudio de las unidades léxicas del campo de la informática e Internet en los diccionarios generales bilingües y en los diccionarios monolingües de aprendizaje pueda mostrarnos, previsiblemente, una visión global del tratamiento que los compiladores de dichas obras confieren a la nomenclatura especializada en general. Asimismo, los datos que se desprendan del análisis de dichas unidades nos proporcionarán una perspectiva más integral que si hubiésemos escogido un campo del saber con menor incidencia interdisciplinar.

Por otro lado, resulta evidente que, en las diferentes fases de la elaboración de los mencionados diccionarios, los avances realizados desde el campo de la informática han transformado no solamente el formato de los mismos, sino también la concepción y el modo de plantear el trabajo lexicográfico. De hecho, esta área de conocimiento influye en todos los niveles de confección de las obras lexicográficas: en su organización, en su estructura, en la distribución de los contenidos, en su fiabilidad y en su accesibilidad.

Por último, consideramos que, analizado un campo de especialidad lo suficientemente representativo y transversal a muchos saberes, el enfoque conferido a dicho campo tiende a menudo a reproducirse en el resto de los campos de especialidad recogidos en los diccionarios. 


\section{El tratamiento del léxico especializado de la informática e Internet: metodología de análisis}

\subsection{La delimitación del campo de aplicación}

Dada la enorme proliferación en el mercado editorial en los últimos años de diccionarios generales bilingües y de diccionarios de aprendizaje, consideramos pertinente, en aras de dotar a nuestro trabajo de una mayor exhaustividad, acotar el campo de aplicación a una serie de obras publicadas por las editoriales más prestigiosas y de uso más extendido en el par de lenguas inglés-español. El principal requisito es que estén en versión electrónica y puedan consultarse en línea mediante una interfaz.

En este sentido, la aplicación del formato electrónico a las obras lexicográficas lleva asociadas una serie de transformaciones, tales como cambios en la distribución de la información, la inclusión de datos multimedia como sonido o imágenes, la posibilidad de realizar múltiples búsquedas empleando operadores booleanos, la incorporación de referencias cruzadas e hipertextos y una mayor accesibilidad (Ortego Antón, 2012: 114).

De este modo, los diccionarios monolingües de aprendizaje que constituyen nuestro campo de aplicación se caracterizan por estar disponibles en línea y presentar una interfaz de consulta sencilla.

1. Merriam Webster's Learner's Dictionary (MWLD), que se centra en la variedad regional de inglés americano, puesto que es uno de los productos del grupo editorial estadounidense líder en la publicación de diccionarios. El contenido se basa en la versión impresa del Merriam Webster's Collegiate Dictionary (11th edition).

Figura 1. Captura de pantalla del $M W L D$.

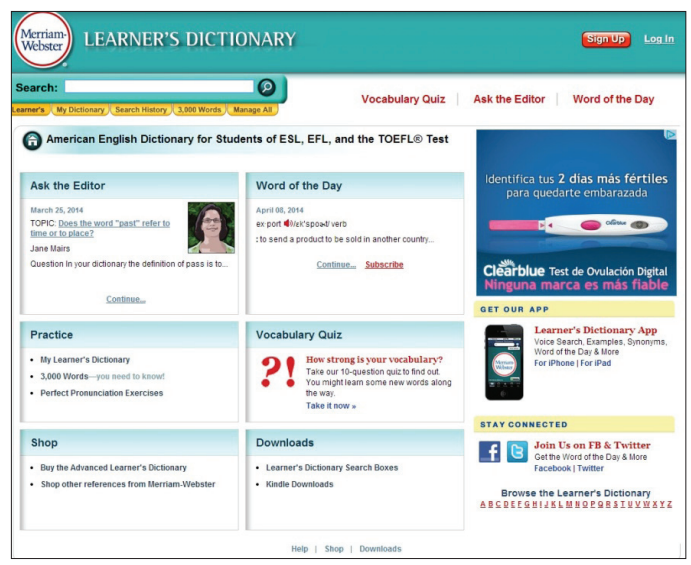


2. Oxford Learner's Dictionaries (OLD), con una interfaz desde la que se ofrece realizar búsquedas tanto en el Oxford Advanced Learner's Dictionary (OALD) si se selecciona en el menú desplegable la opción «English», como en el Oxford Advanced American Dictionary (OAAD) si se escoge la opción «American». Las definiciones se caracterizan por utilizar únicamente 3000 palabras (The Oxford $\left.3000^{T M}\right)$, que fueron escogidas cuidadosamente por expertos lingüísticos y profesores basándose en dos criterios: la relevancia y la frecuencia de uso en dos corpus (British National Corpus y Oxford Corpus Collection).

Figura 2. Captura de pantalla del OLD.

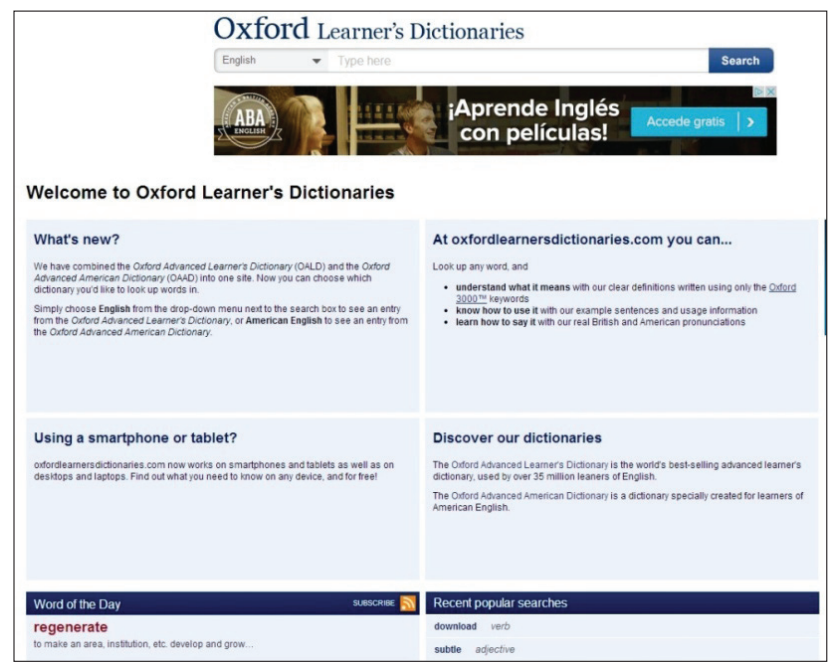

3. Cambridge Advanced Learner's Dictionary (CALD), que presenta una interfaz que permite realizar búsquedas tanto en todos los diccionarios de esta editorial como únicamente en el diccionario de aprendizaje. Además, en el apartado denominado «key features» de la interfaz se explican las características propias de esta obra, entre las que destacan el empleo de definiciones utilizando un lenguaje claro y simple, así como la descripción de ejemplos de uso, que indican el uso de una unidad léxica en un contexto real. 
Figura 3. Captura de pantalla del CALD.

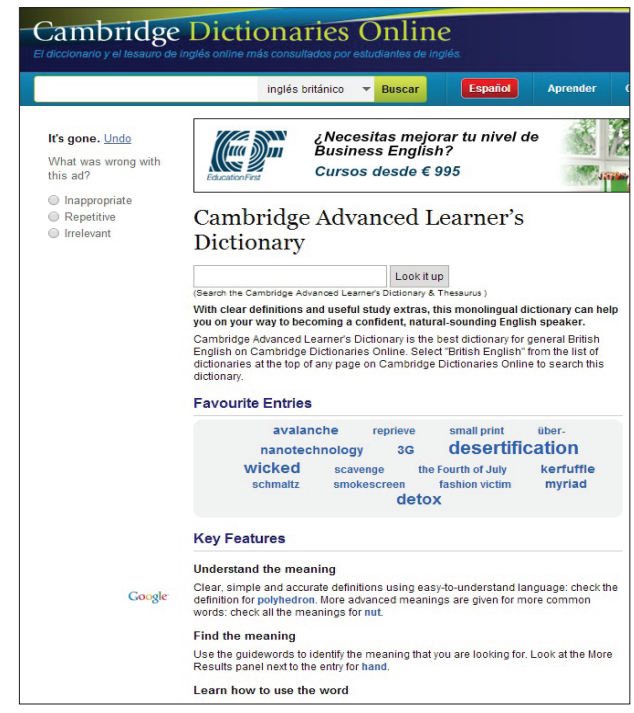

4. Longman Dictionary of Contemporary English (LDOCE), cuya descripción se ofrece en la pestaña «about LDOCE». Contiene más de 155000 ejemplos. Para elaborar las definiciones los compiladores han utilizado un repertorio de 2000 palabras.

Figura 4. Captura de pantalla del LDOCE.

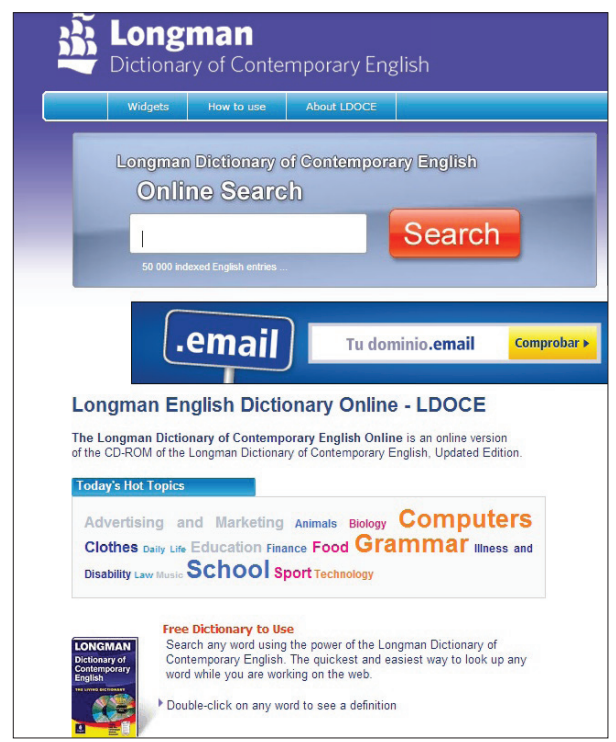


5. Collins English for Learners (COBUILD) se caracteriza por la simplicidad de su interfaz y por no incluir publicidad, lo que ayuda al estudiante a no distraer la atención.

Figura 5. Captura de pantalla del COBUILD.

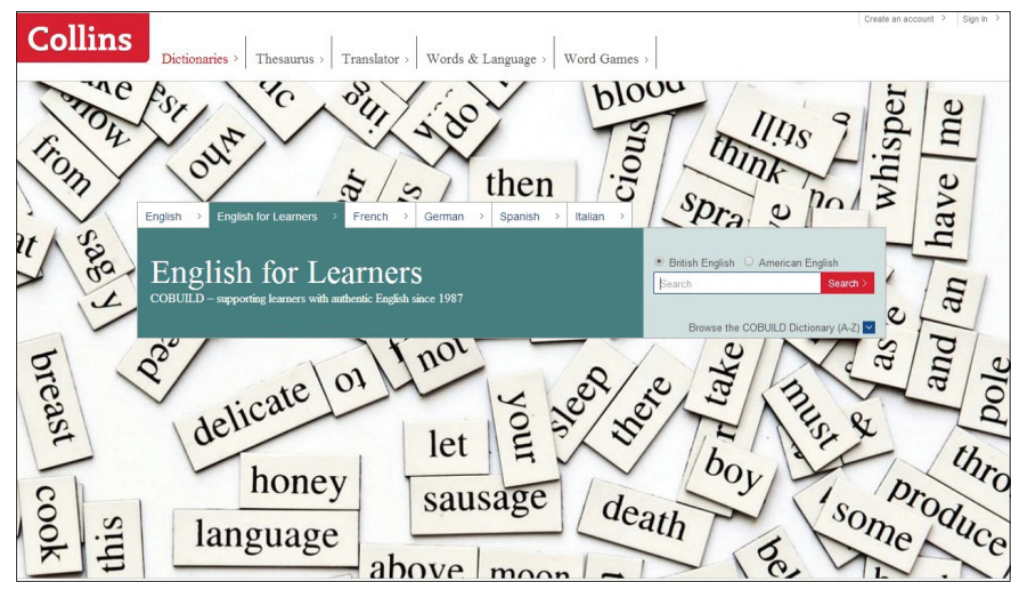

Por lo que respecta a la selección de los diccionarios generales bilingües, hemos procedido a elegir cuatro obras disponibles en línea basándonos en parámetros como el uso y el prestigio de las editoriales.

1. Cambridge inglés-español (CIE), que comparte la misma interfaz que el $C A L D$, dado que en una pestaña de dicha interfaz el usuario elige el diccionario en el que desea realizar la búsqueda.

Figura 6. Captura de pantalla del CIE.

\begin{tabular}{|c|c|c|}
\hline \multicolumn{3}{|c|}{ 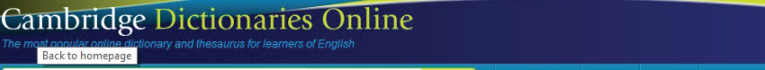 } \\
\hline \multicolumn{2}{|c|}{ Endish-Sparish Search! } & Lean Share Develop \\
\hline \multirow{2}{*}{$\begin{array}{l}\text { It's gone. Unde } \\
\text { What was wong with } \\
\text { this ad? }\end{array}$} & \multirow{2}{*}{\multicolumn{2}{|c|}{ 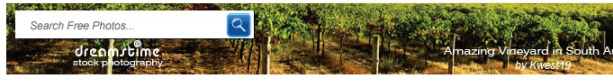 }} \\
\hline & & \\
\hline \multirow{11}{*}{$\begin{array}{l}\text { Inappropriate } \\
\text { Repetitive } \\
\text { Irrelevant }\end{array}$} & Diccionario Password inglés-español & \multirow{11}{*}{ 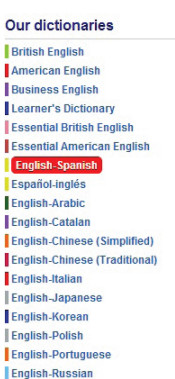 } \\
\hline & \begin{tabular}{|l|l|l|} 
Translate rom Spanish to English & Español-Inglés & Inglés-Espaniol \\
\end{tabular} & \\
\hline & 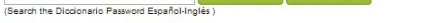 & \\
\hline & View this page in Engllsh & \\
\hline & $\begin{array}{l}\text { Este dilccionario blingue español-nglés/nglés-español orrece una amplla } \\
\text { cobortura del inglós. }\end{array}$ & \\
\hline & $\begin{array}{l}\text { - más de } 35.000 \text { eniradas } \\
\text { - defininicones breves y sencillos en inglés con traducciones en español } \\
\text { numerosos ejemplos de uso }\end{array}$ & \\
\hline & 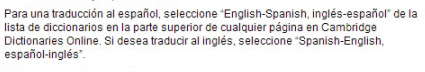 & \\
\hline & Būsquedas favoritas & \\
\hline & carol connect & \\
\hline & fleece friend island listen lurch nape & \\
\hline & $\begin{array}{cccc}\text { ombudsman } & \begin{array}{c}\text { self-assurance } \\
\text { nino }\end{array} & \begin{array}{c}\text { side } \\
\text { carta }\end{array} \text { niña } & \text { where } \\
\text { deshielo } & \text { encantador }\end{array}$ & \\
\hline
\end{tabular}


2. WordReference (WR), obra que muestra los resultados obtenidos tanto en su propio diccionario como en el diccionario Collins Spanish Dictionary, en función de la pestaña que se active durante la búsqueda.

Figura 7. Captura de pantalla del WR.

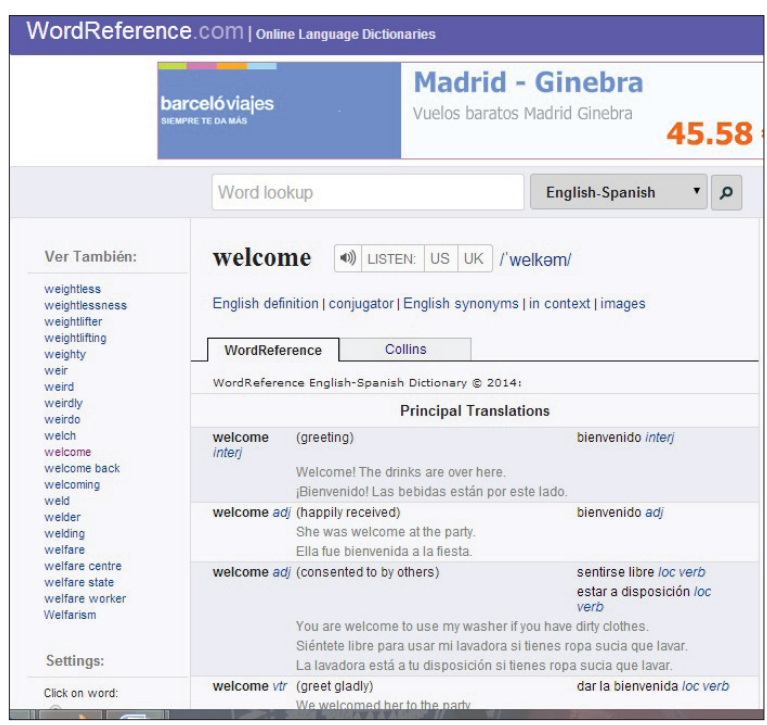

3. English-Spanish Oxford Dictionary (ESOD) se considera uno de los diccionarios generales bilingües más completos porque ofrece cobertura a 800000 palabras, frases y traducciones. Además, se complementa con otros recursos que asisten en el aprendizaje de lenguas y durante el proceso de traducción.

Figura 8. Captura de pantalla del ESOD.

\begin{tabular}{|l} 
(1) Oxford Dictionaries \\
Language motters
\end{tabular}


4. Collins English-Spanish Dictionary (CESD), diccionario en el que volvemos a constatar que la interfaz es semejante a la utilizada en el diccionario de aprendizaje COBUILD, dado que según se seleccione una u otra pestaña, la búsqueda se realiza en distintos diccionarios.

Figura 9. Captura de pantalla del CESD.

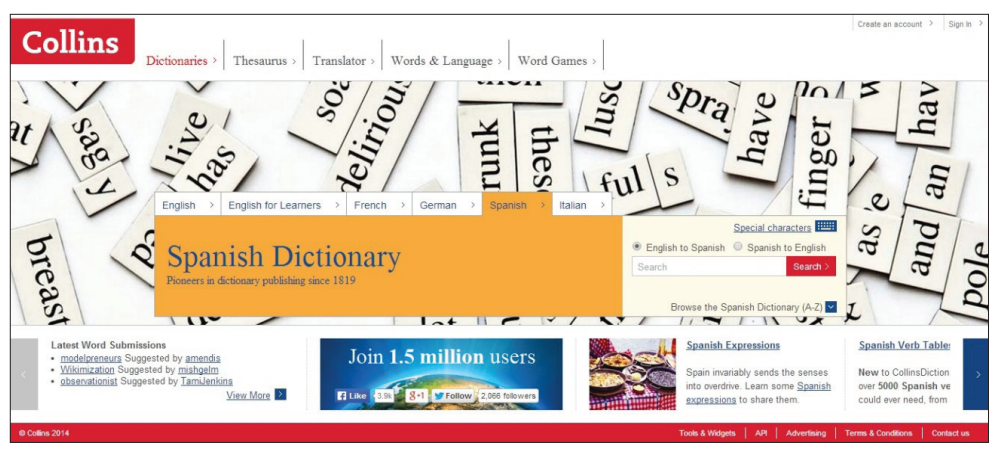

\subsection{Parámetros de análisis}

Una vez delimitado el campo de aplicación, procedemos a realizar un análisis en forma de cata siguiendo la metodología empleada en trabajos previos (Roberts, 2004; Josselin, 2005).

Las unidades que forman parte de la muestra han sido extraídas de un corpus ad hoc. Dicho corpus se compone de 11 textos publicados de 2009 a 2014 que han sido seleccionado aleatoriamente a través de una búsqueda avanzada con Google.

Los textos se caracterizan por ser de acceso libre, por estar destinados a aprendices de lenguas con un nivel B1 de inglés según el MCER (Consejo de Europa, 2001) y por emplearse para actividades de comprensión escrita, es decir, para poner en práctica la destreza de reading para la preparación del examen PET de Cambridge University Press. Seis de los textos proceden de portales web destinados al aprendizaje de la lengua inglesa: BBC Learning English ${ }^{1}$, Exam English ${ }^{2}$ o Using English ${ }^{3}$. El resto de los textos proceden de la prensa escrita inglesa, han sido adaptados y se han utilizado en los últimos cinco años en los exámenes de PET.

$1<$ http://www.bbc.co.uk/learningenglish/> (Consulta: 08/12/2014).

$2<$ http://www.examenglish.com/PET/index.html> (Consulta: 08/12/2014).

$3<$ http://www.usingenglish.com/> (Consulta: 08/12/2014). 
Para seleccionarlos hemos procedido a realizar una búsqueda avanzada con Google limitando los resultados sitios web destinados al aprendizaje del inglés y a los exámenes empleados en la prueba de PET. A continuación, hemos escrutinado los resultados y hemos seleccionado únicamente aquellos textos que en el título incluyesen unidades léxicas que hiciesen referencia a conceptos del campo de la informática e Internet. En total, nuestro corpus ad hoc contiene 8202 tipos y 1954 ocurrencias.

Con la ayuda del analizador de concordancias AntConc realizamos un vaciado de las palabras clave más frecuentes comparando los resultados de nuestro corpus con los obtenidos en un corpus de referencia de la lengua inglesa, el LancasterOslo-Bergen Corpus (LOB).

Del listado de unidades más frecuentes ofrecido por AntConc eliminamos las unidades que consideramos que pertenecen al léxico general (ej.: user) así como los nombres propios (ej.: Facebook), de manera que las unidades léxicas más frecuentes que constituirán nuestra muestra de análisis se enumeran a continuación:

1. technology

2. computer

3. internet

4. online

5. access

6. social networking

7. IT

8. software

9. mobile phone

10. smartphone

Por lo que respecta a los parámetros que tendremos en cuenta para realizar el análisis, en primer lugar comprobaremos si la unidad léxica se incluye en la nomenclatura de los diccionarios que constituyen nuestro campo de aplicación. Además, constataremos si se marca diatécnicamente empleando algún tipo de mecanismo y qué definición o equivalente se propone. A continuación, verificaremos si la definición o el equivalente se acompaña de información contextual, por ejemplo actantes, colocaciones o ejemplos de uso, y si se incorpora al contenido del artículo lexicográfico algún tipo de información que pueda resultar relevante para los estudiantes de lengua extranjera en tareas tanto de codificación como de descodificación.

Por último, procederemos a contrastar los resultados obtenidos entre obras de una misma tipología así como entre las dos tipologías escogidas. 


\title{
5. Análisis y resultados
}

\section{$5.1 M W L D$}

Excepto tres unidades léxicas, las relativas a «social networking», «IT» y «smartphone», el resto de la muestra de unidades se recogen como entradas en la nomenclatura. Generalmente el lema se presenta con división silábica y se acompaña de la pronunciación con el símbolo de un altavoz, que permite escuchar dicha pronunciación pulsando en el mismo, y con la transcripción fonética. También se incluyen diversas acepciones en las que se ofrece una definición en lengua inglesa, la categoría gramatical, la posición de la unidad en el sintagma, posibles colocaciones de dicha unidad y varios ejemplos de uso, tal y como puede comprobarse a modo de ejemplo en la entrada relativa a «Internet»:

Figura 10. Fragmento de la entrada de «Internet» en el $M W L D$.

\author{
Internet \\ 2 entries found for internet. \\ Internet (noun) \\ Internet service provider (noun) \\ In-ter-net w) /'intz, net/ noun \\ Meaning: \\ the Internet \\ : a system that connects computers throughout the world \\ - She spent hours surfing the Internet. - compare INTRANET, WORLD WIDE WEE \\ - Internet adjective always used before a noun \\ - an Internet connection - an Internet site
}

Por otro lado, hemos constatado que la unidad «IT» no se recoge en la nomenclatura, ni tampoco la denominación completa a la que hace referencia dicha sigla (information technology), fenómeno similar al detectado en el caso de las unidades «social networking» $y$ «smartphone».

Además, nos gustaría señalar que en la entrada relativa a «mobile phone» únicamente se ofrece una referencia cruzada a otra entrada, «cell phone».

\section{$5.2 \mathrm{OLD}$}

En esta obra hemos corroborado que todas las unidades de la muestra de análisis se recogen como entradas en la nomenclatura. En el contenido del artículo lexico- 
gráfico se ofrece la categoría gramatical, las variantes gráficas si las hubiera, la pronunciación con las diversas variantes regionales de inglés británico e inglés americano (pueden escucharse si se pulsa en el altavoz), su uso más frecuente, otras posibilidades de empleo de dicha unidad según el registro, la definición y varios ejemplos de uso en los que se muestran las colocaciones de la unidad. Además, ciertas unidades van acompañadas de una nota de uso en la que se presentan los actantes más utilizados que pueden dar problemas a los aprendices de lenguas.

\section{Figura 11. Fragmento de la entrada de «Internet» en el OLD.}

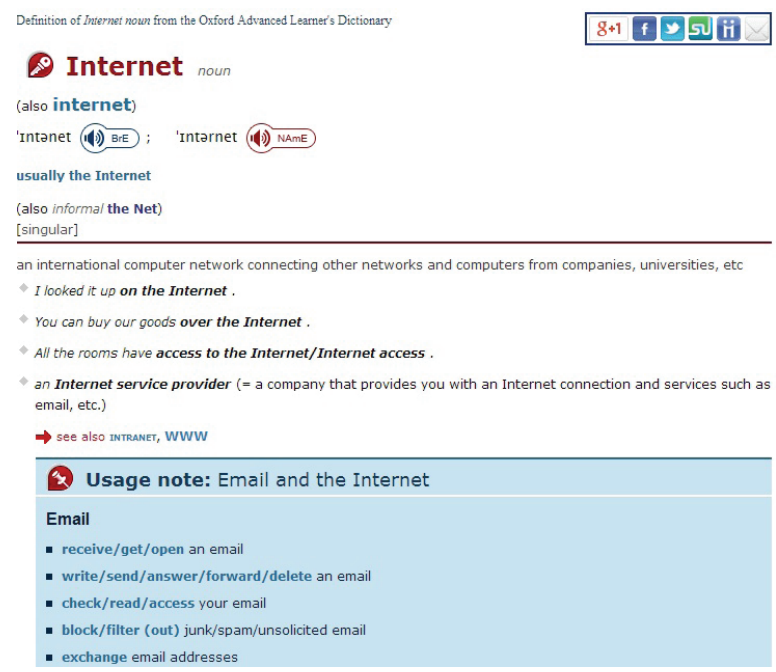

\subsection{CALD}

Salvo la unidad «IT», el resto de las unidades de la muestra se recogen en la nomenclatura. No obstante, hemos comprobado que «information technology» se incluye en la nomenclatura y como abreviatura de dicha unidad se ofrece «IT».

Respecto al contenido del artículo lexicográfico, se ofrece la unidad por defecto acompañada del artículo «the», la categoría gramatical, el número, dos altavoces, uno en cada color, para poder escuchar la pronunciación en inglés británico o en inglés americano seguido de la correspondiente transcripción fonética en cada variedad regional y la variante de registro en lengua informal entre paréntesis.

A continuación, se ofrece una etiqueta, A1, que se refiere al nivel en que dicha unidad debe ser aprendida según los distintos niveles propuestos por el MCER (Consejo de Europa, 2001). Además, se recoge una definición, un ejemplo de uso y varias notas gramaticales que pueden ser de interés para los aprendices de lenguas, dado que van enfocadas a la producción de mensajes en este medio. 
Figura 12. Fragmento de la entrada de «Internet» en el CALD.

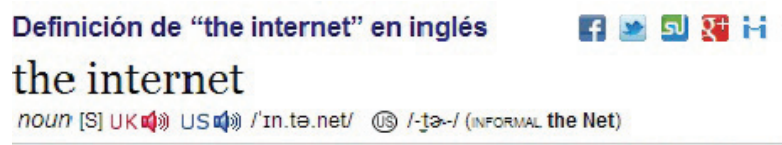

(Definition of the internet noun from the Cambridge Advanced Learner's Dictionary \&. Thesaurus a Cambridge University Press)

\subsection{LDOCE}

Todas las unidades de la muestra salvo una, «social networking», están incluidas en la nomenclatura de este diccionario. No obstante, nos gustaría precisar que en los resultados de búsqueda la interfaz ofrece todos los lemas que contienen la unidad introducida en la casilla de búsqueda. Por ejemplo, si buscamos «Internet», los resultados obtenidos se muestran en la siguiente captura de pantalla:

Figura 13. Resultados de la búsqueda de «Internet» en el LDOCE.

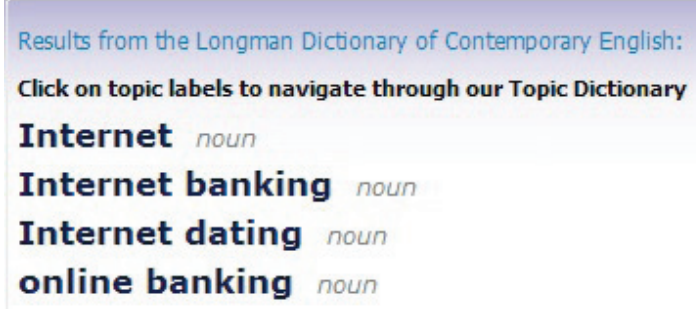

En consecuencia, elegimos la unidad deseada, la primera, que hace referencia a «Internet». En el contenido del artículo lexicográfico se registra el lema con la categoría gramatical, un altavoz que al hacer clic sobre él nos permite escuchar la pronunciación, de nuevo el lema con la separación silábica, posibles variantes gráficas, la unidad con el artículo seguida de la definición, la colocación más frecuente y varios ejemplos de uso que también están disponibles con la transcripción oral. 
A continuación, en un recuadro se muestran colocaciones y unidades relacionadas con la unidad seleccionada y referencias cruzadas a términos del mismo campo semántico.

Figura 14. Fragmento de la entrada de «Internet» en el LDOCE.

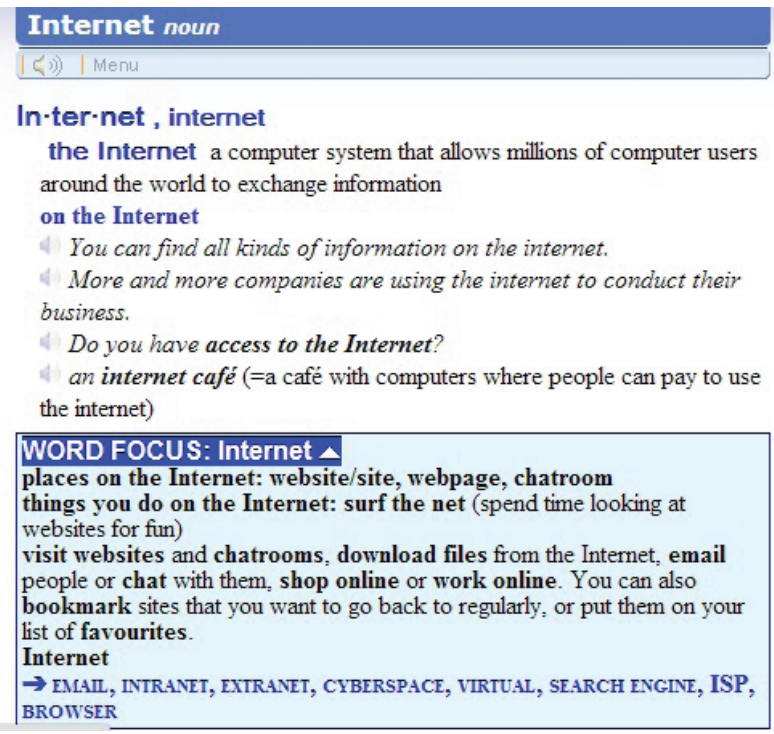

\subsection{COBUILD}

En primer lugar, la interfaz nos pide que elijamos la variante regional en la que deseamos realizar la búsqueda, inglés británico o inglés americano. Optamos por inglés británico y constatamos que todas las unidades de la muestra salvo una, «smartphone», se incluyen en la nomenclatura de esta obra.

En el artículo lexicográfico se presenta el lema, la transcripción fonética, un altavoz que permite escuchar la pronunciación y una variante gráfica. Seguidamente se ofrece la definición de la unidad y el equivalente de traducción en multitud de lenguas.

Sin embargo, constatamos que no hay ejemplos de uso ni información que nos permita utilizar esta unidad en contexto. 
Figura 15. Fragmento de la entrada de «Internet» en el COBUILD.

\begin{tabular}{|c|c|}
\hline \multicolumn{2}{|l|}{ Definitions } \\
\hline \multicolumn{2}{|c|}{$\begin{array}{l}\text { The Internet is the computer network which allows computer users to connect with computers all over } \\
\text { the world, and which carries e-mail. }\end{array}$} \\
\hline \multicolumn{2}{|c|}{ - Translations for 'Internet' } \\
\hline \multicolumn{2}{|c|}{$\begin{array}{l}\text { British English: Internet The Internet is the computer network which allows computer users to } \\
\text { connect with computers all over the world, and which carries email. Inte net NOUN }\end{array}$} \\
\hline Arabic: & Brazilian Portuguese: Internet \\
\hline Chinese: 因特网 槠明 & Croatian: internet \\
\hline Czech: internet & Danish: internet \\
\hline Dutch: internet & European Spanish: Internet \\
\hline Finnish: internet & French: Internet \\
\hline German: Internet & Greek: бієס̄ikтuo (1) \\
\hline Italian: Internet & Japanese: インターネット 唹 \\
\hline Korean: 인터넷 때키 & Norwegian: Internett \\
\hline Polish: Internet & Portuguese: Internet \\
\hline Romanian: internet & Russian: Интернет (1) \\
\hline Snanish- Internet & Swedish-Internet \\
\hline
\end{tabular}

\subsection{CIE}

En esta obra se recogen en la nomenclatura todas las unidades salvo «IT», que no aparece como entrada ni se ofrece dicha unidad como abreviatura de «information technology». En primer lugar se ofrece el lema, la categoría gramatical, la transcripción fonética en inglés británico y, si hubiera variantes, en inglés americano. A continuación, se presenta una breve descripción que coincide con la proporcionada en el CALD, seguida de los equivalentes, que proceden del Password English/Spanish Dictionary, alguna colocación y ejemplos de uso en lengua inglesa sin traducir.

Nos gustaría reseñar que en las entradas conferidas a «social networking», «mobile phone»y «smartphone», el diccionario ofrece la misma información que la ofrecida en dichas entradas en el CALD, por lo que se omite el equivalente de traducción, a pesar de que esta obra se caracteriza por ser un diccionario general bilingüe.

Además, observamos que no existen datos relativos al marcado diatécnico de estas unidades. 
Figura 16. Fragmento de la entrada de «Internet» en el CIE.

\section{Spanish translation of "Internet" $\quad$ 대 파 $\mathrm{i}-\mathrm{i}$ Internet noun /'intenet, (American) 'internet/}

$\rightarrow$ a worldwide computer network that provides information on very many subjects and enables users to exchange messages.

Internet

an internet cafe

Do you have access to the internet?

(Definition of Internet from the Password English-Spanish Dictionary (9) $2013 \mathrm{~K}$ Dictionaries Ltd)

\section{$5.7 W R$}

En este diccionario se registran todas las unidades de la muestra salvo «IT».

En primer lugar se ofrece el lema, tras el cual se ofrece la transcripción fonética y varias acepciones con la categoría gramatical en abreviatura. Asimismo, se indica la zona de uso y entre paréntesis una breve definición, tras la cual se ofrecen los equivalentes de traducción y varios ejemplos de uso acompañados de su traducción. Además, en la parte inferior de la pantalla se ofrecen unidades compuestas.

Por lo general, en esta obra no se suelen incluir etiquetas para indicar el campo del saber al que pertenece una unidad especializada, aunque en ciertas ocasiones, como ocurre con la unidad «online», entre paréntesis y con cursiva se registra «Internet». 
Figura 17. Fragmento de la entrada de «Internet» en el WR.

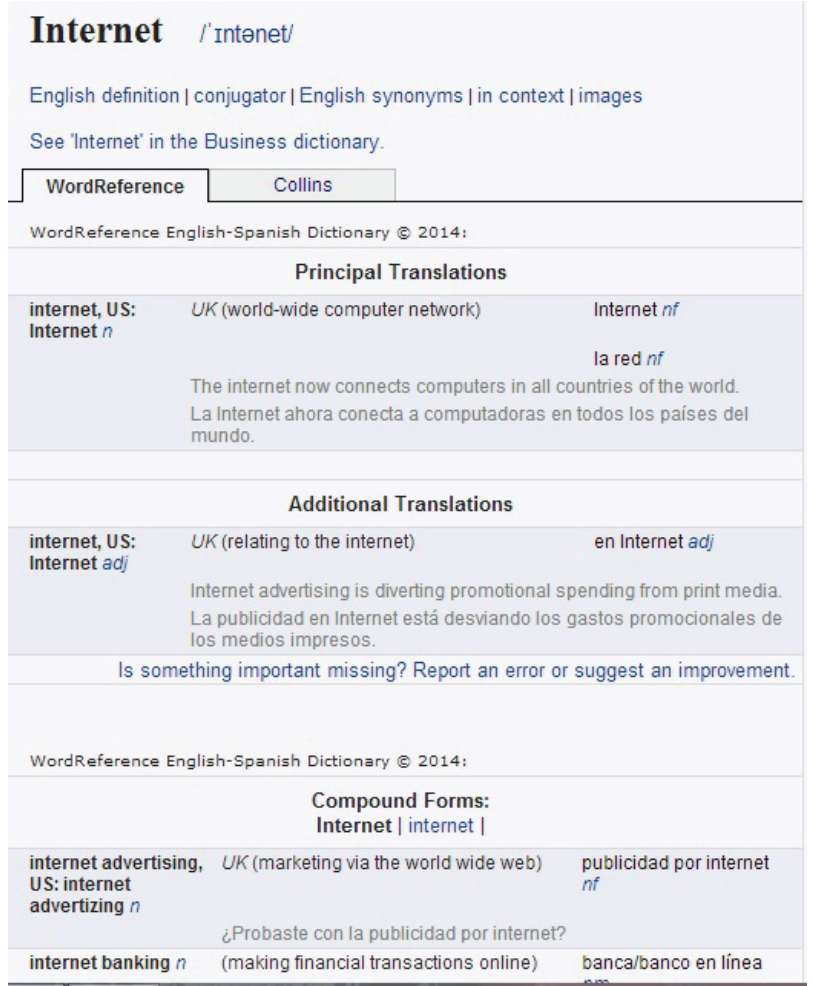

\subsection{ESOD}

Este diccionario recoge todas las unidades de la muestra salvo «IT». Tal y como hemos constatado en varios de los diccionarios analizados, la sigla tampoco se incluye dentro de la entrada conferida a «information technology».

Respecto al artículo lexicográfico, se recoge el lema, la pronunciación, la categoría gramatical y dos etiquetas en mayúscula y entre corchetes que hacen referencia al campo del saber en el que se emplea la unidad. En el caso de «Internet», esta unidad se emplea en informática «[COMPUT]» y en economía «[ECON]». A continuación, se indica el número y el equivalente de traducción, seguido de varias colocaciones y ejemplos de uso en inglés y en español. Por último, se recogen una serie de unidades léxicas compuestas con sus traducciones al español. 
Figura 18. Fragmento de la entrada de «Internet» en el ESOD.

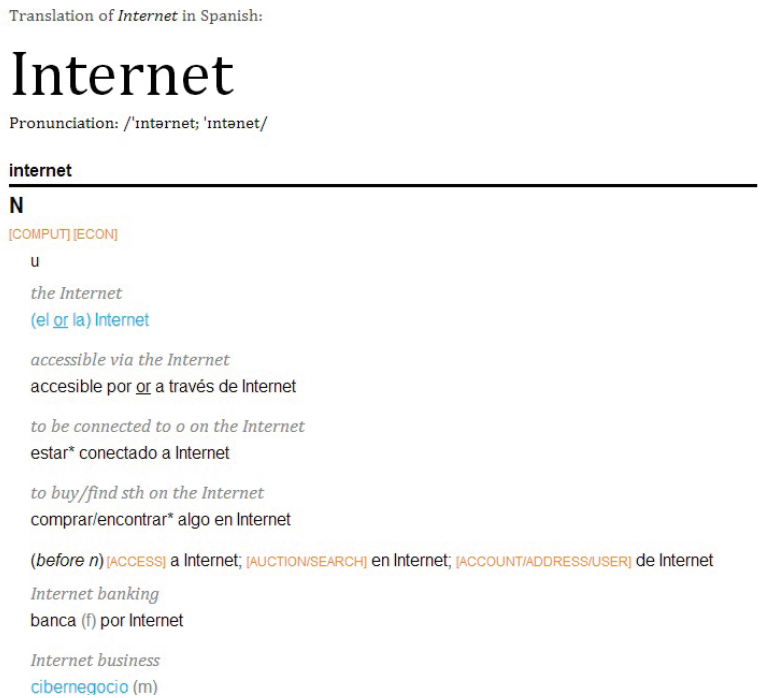

Por lo que respecta al resto de unidades analizadas en esta obra, tres de ellas están marcadas con "[COMPUT]», que son las relativas a «computer», «online» $y$ «access», una de ellas, «Internet», se registra con dos marcas, tal y como hemos mencionado previamente, así como otras dos unidades, «mobile phone» $y$ «smartphone», que se incluyen con la etiqueta «[TELEC]».

\subsection{CESD}

En este diccionario se recogen todas las unidades de la muestra. Se presenta el lema, seguido de la transcripción fonética y del símbolo de un altavoz para escuchar la pronunciación. Seguidamente se ofrece la categoría gramatical y los equivalentes de traducción, con varios actantes entre corchetes y una serie de compuestos. Sin embargo, observamos que no se ofrecen ejemplos de uso.

Además, constatamos que ciertas unidades se incluyen con marcado diatécnico, por ejemplo «online», «access», «IT», «software» $y$ «smartphone» se incluyen con la etiqueta «(Computing)». 
Figura 19. Fragmento de la entrada de «Internet» en el CESD.

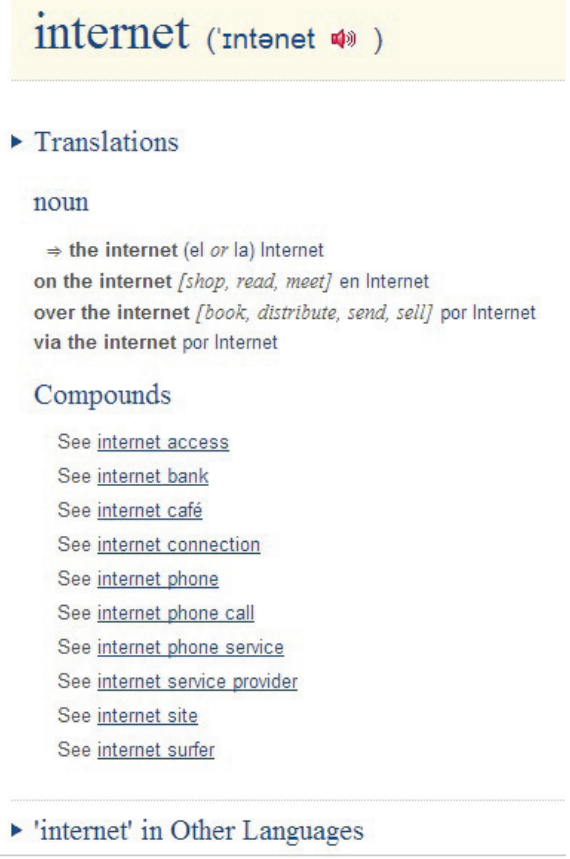

\subsection{Comparación y resultados}

Por un lado, observamos que todas las obras han introducido nuevas funciones que van asociadas al formato electrónico, por ejemplo la posibilidad de escuchar la pronunciación de la unidad haciendo clic en el símbolo del altavoz.

Sin embargo, por lo que respecta al marcado diatécnico, constatamos que la tendencia general empleada en los diccionarios de aprendizaje es la ausencia de utilización de etiquetas para indicar la pertenencia de una unidad léxica a un determinado campo del saber. Además, la omisión de marca diatécnica también se pone de manifiesto en algunos de los diccionarios generales bilingües, por ejemplo en el CIE y en el WR.

Asimismo, hemos comprobado que existen diferencias respecto de la información ofrecida. Por ejemplo, hemos contemplado que varios de los diccionarios de aprendizaje están orientados a una variedad regional de la lengua inglesa: inglés británico o inglés americano. En este sentido, el MWLD ofrece cobertura a esta última variedad y el COBUILD permite seleccionar la variedad en la que realizar la búsqueda. 
Además, hemos contrastado que en ocasiones las definiciones o los equivalentes de traducción van desprovistos de información contextual, tal y como ocurre en el COBUILD. De hecho, los editores de esta obra y del CESD ofrecen la misma información, ya se trate de una búsqueda en el diccionario de aprendizaje o en el diccionario general bilingüe.

Por último, el tratamiento de los equivalentes ofrecidos en los diccionarios generales bilingües varía en función de la obra que se consulte, como puede constatarse en la síntesis que presentamos en la siguiente tabla:

Tabla 1. Equivalentes ofrecidos en los diccionarios bilingües.

\begin{tabular}{|c|c|c|c|c|}
\hline $\begin{array}{l}\text { UNIDAD } \\
\text { LÉXICA }\end{array}$ & CIE & $W R$ & ESOD & CESD \\
\hline technology & tecnología & tecnología & tecnología & tecnología \\
\hline computer & ordenador & $\begin{array}{l}\text { ordenador, } \\
\text { computadora }\end{array}$ & $\begin{array}{c}\text { computadora, } \\
\text { computador, ordenador }\end{array}$ & $\begin{array}{l}\text { ordenador, computador, } \\
\text { computadora }\end{array}$ \\
\hline Internet & Internet & Internet & internet & Internet \\
\hline online & online & por internet, online & en línea, conectado & online, en línea, conectado \\
\hline access & acceso & $\begin{array}{l}\text { acceso, acceder, } \\
\text { entrar en }\end{array}$ & acceso a algo & acceso, conseguir acceso a \\
\hline $\begin{array}{c}\text { social } \\
\text { networking }\end{array}$ & $\mathrm{NO}$ & contactos por la red & & sitio de redes sociales \\
\hline$I T$ & & & & informática \\
\hline software & software & $\begin{array}{l}\text { software (voz } \\
\text { inglesa) }\end{array}$ & software & software \\
\hline mobile phone & $\mathrm{NO}$ & móvil & teléfono celular/móvil & teléfono móvil, celular \\
\hline smart phone & $\mathrm{NO}$ & $\begin{array}{l}\text { smartphone / } \\
\text { teléfono inteligente }\end{array}$ & teléfono inteligente & $\begin{array}{l}\text { teléfono inteligente, } \\
\text { smartphone }\end{array}$ \\
\hline
\end{tabular}

En consecuencia, los usuarios encontrarán información diferente de una misma unidad según la obra que consulten. Esta ausencia de sistematicidad podría superarse si en los diccionarios generales bilingües se ofrecieran etiquetas en las que se indicara el contexto de uso de cada uno de los equivalentes propuestos, de manera que el usuario se viese asistido en su elección.

\section{Conclusiones}

A la vista de los resultados obtenidos, observamos la heterogeneidad de las informaciones registradas en los artículos lexicográficos de las principales herramientas a las que acuden los estudiantes de lenguas extranjeras. 
Por un lado, hemos comprobado que los patrones de presentación de la información sobre el uso de una unidad léxica varía entre las obras consultadas, que el marcado diatécnico no se lleva a cabo en los diccionarios de aprendizaje y que los equivalentes de los diccionarios generales bilingües se caracterizan por la amplia variedad de posibilidades.

Además, en ninguna de las obras consultadas se registran datos relativos a cómo utilizar las unidades en un determinado contexto. Conscientes de la importancia del aprendizaje de las lenguas en la sociedad actual, así como de la competencia documental durante la codificación y la descodificación que se produce durante la comunicación interlingüística, nuestra tarea como docentes de lenguas extranjeras se corresponde con formar a los estudiantes en el uso de los diccionarios. Por tanto, uno de nuestros cometidos será mostrar las virtudes y los defectos de cada una de las tipologías y de las obras analizadas, de manera que los estudiantes sean capaces de seleccionar la obra que mejor satisfaga sus necesidades.

Por último, somos optimistas y consideramos que llegará el día en que los avances de la lingüística de corpus y de la lexicografía se incorporen a las nuevas ediciones de los diccionarios electrónicos y, de este modo, se implemente la información en ellos registrada, ofreciéndose un uso real de la lengua.

\section{Referencias bibliográficas}

\subsection{Diccionarios}

\subsubsection{Diccionarios monolingües de aprendizaje}

Cambridge Advanced Learner's Dictionary (CALD) $[<$ http://dictionary.cambridge.org/es/diccionario/britanico/>) (Consulta: 15/09/2014).

Collins English for Learners (COBUILD) [<http://www.collinsdictionary.com/ dictionary/english-cobuild-learners $>$ ] (Consulta: 15/09/2014).

Longman Dictionary of Contemporary English (LDOCE) [<http://www.ldoceonline.com/>] (Consulta: 15/09/2014).

Merriam Webster's Learner's Dictionary (MWLD) [<http://www.learnersdictionary.com/>] (Consulta: 15/09/2014).

Oxford Learner's Dictionaries (OLD) $[<$ http://www.oxfordlearnersdictionaries. $\mathrm{com} />$ ] (Consulta: 15/09/2014).

\subsubsection{Diccionarios generales bilingües}

Cambridge inglés-español (CIE) [<https://dictionary.cambridge.org/dictionary/ english-spanish/>) (Consulta: 15/09/2014). 
Collins English-Spanish Dictionary (CESD) [<http://www.collinsdictionary. com/dictionary/english-spanish>] (Consulta: 15/09/2014).

English-Spanish Oxford Dictionary (ESOD) [<http://www.oxforddictionaries. com/spanish/>] (Consulta: 15/09/2014).

WordReference (WR) [<http://www.wordreference.com/>] (Consulta: 15/09/2014).

\subsection{Otros}

BÉjoint, H. (1981). «The Foreign Student's Use of Monolingual English Dictionaries. A Study of Language Needs and Reference Skills». Applied Linguistics, 2(3): 207-222.

Bergenholtz, H. y S. Tarp (2002). «Die moderne lexicographische Funktionslehre Diskussionsbeitrag zu neuen und alten Paradigmen, die Wörterbüchen als Gebrauchagegen stände verstehen». Lexicographica, 18: 253-263.

Consejo de Europa (2001). Marco Común Europeo de Referencia para las Lenguas. Estrasburgo: Consejo de Europa.

Cowie, A. P. (1999). English Dictionaries for Foreigh Learners - A History. Oxford: Clarendon Press.

Fuertes Olivera, P.y A. Arribas Baño (2008). Pedagogical Specialized Lexicography: the Representation of Meaning in English and Spanish Business Dictionaries. Amsterdam/Philadelphia: John Benjamins.

Granger, S. (1994). «The Learner Corpus: a Revolution in Applied Linguistics». English Today, 39(3): 25-29.

Granger, S. y M. Paquot (2010). Elexicography in the 21st century: new challenges, new applications, Cabiers du Cental, 7. Louvain-la-Neuve: Presses Universitaires de Louvain.

Gutiérrez Rodilla, B. (1998). La ciencia empieza en la palabra. Análisis e historia del lenguaje científico. Barcelona: Península.

Hartmann, R. R. K. (1989). «The Dictionary as an Aid to Foreign Language Teaching». WDD, 5(1): 102-111.

Hartmann, R. R. K. y G. James (1998). Dictionary of Lexicography. London: Routledge.

ILson, R. (1999). «Nine Learner's Dictionaries». International Journal of Lexicography, 12(3): 223-237.

Josselin, A. (2005). Place et rôle des terminologies dans les dictionnaires généraux unilingues et bilingues. Étude d'un domaine de spécialité: Volcanologie. Tesis doctoral. Lyon: Université Lumière Lyon II. Centre de Recherche en Termi- 
nologie et Traduction [en línea]. [<http://theses.univ-lyon2.fr/documents/ lyon $2 / 2005 /$ josselin_a\#p $=08 \mathrm{a}=$ top $>$ ].

Landau, S. I. (2001). Dictionaries: The Art and Craft of Lexicography. 2a Ed. Cambridge: Cambridge University Press.

McArthur, T. (1998). The English Languages. Cambridge: Cambridge University Press.

NesI, H. (1999). "A user's guide to electronic dictionaries for language learners». International Journal of Lexicography, 12(1):55-66.

- (2000). The Use and Abuse of EFL Dictionaries. Tubingen: Max Niemeyer Verlag.

Ortego Antón, M. T. (2012). Estudio contrastivo inglés/español del tratamiento del léxico especializado recogido en los diccionarios generales bilingües $\mathrm{CU} y$ GDO. Tesis doctoral. Valladolid: Universidad de Valladolid [<https://uvadoc.uva.es $/$ handle $/ 10324 / 41 /$ browse?type $=$ author\&order $=$ ASC\&rpp $=20$ $\&$ value $=$ Ortego + Ant $\%$ C3\%B3n\%2C + Mar\%C3\%ADa+Teresa $>$ ].

Rodríguez Reina, M. P. (2002). «Las marcas de especialidad: Una cuenta pendiente de difícil solución». En: G. Guerrero Ramos, y M. F. Pérez Lagos (eds.). Panorama actual de la terminología. Granada: Comares, 327-357.

Roberts, R. P. (2004). «Terms in General Dictionaries ». En: J. M. Bravo Gozalo (ed.). A New Spectrum of Translation Studies. Valladolid: Universidad de Valladolid, 121-140.

Stein, G. (2002). Better Words: Evaluating EFL Dictionaries. Exeter: Exeter University Press.

TArp, S. (2006). «Lexicografía de aprendizaje». Cadernos de Tradução, 18(2): $295-317$.

Thoiron, P. (1998). «Place et rôle de la terminologie dans les dictionnaires bilingues non spécialisés. Le cas de la terminologie médicale dans le Dictionnaire Hachette-Oxford (français-anglais)». En: S. Mellet y M. Vuillaume (eds.): Mots chiffrés et déchiffrés. Paris: Honoré Champion, 621-650.

Tribble, C. (2003). «Five Electronic Learner's Dictionaries». ELT Journal, 52(2): 182-197.

Tono, Y. (1996). «Using learner corpora for L2 lexicography: information of collocational errors for EFL learners». Lexikos, 6: 116-132. 